\title{
Liver and Intrahepatic Bile Duct Benign Non-Epithelial Neoplasm
}

National Cancer Institute

\section{Source}

National Cancer Institute. Liver and Intrahepatic Bile Duct Benign Non-Epithelial

Neoplasm. NCl Thesaurus. Code C5749.

A benign non-epithelial neoplasm that affects the liver parenchyma and intrahepatic bile ducts. Representative examples include hemangioma, angiomyolipoma, and solitary fibrous tumor. 